\title{
Clique Communities in Social Networks
}

Luís Cavique, Universidade Aberta, Portugal, Icavique@univ-ab.pt Armando B. Mendes, Universidade Açores, Portugal, amendes@uac.pt Jorge M.A. Santos, Universidade Évora, Portugal, jmas@uevora.pt

\section{Introduction}

After Tim Berners-Lee's (2006) communication on the three ages of the Web in the International World Wide Web Conference WWW2006, there has been an explosion of interest in the social networks associated with Web 2.0 in an attempt to improve socializing and come up with a new model for knowledge management. Even though Tim Berners-Lee had imagined a readand-write Web, the Web was originally a read-only medium for the majority of the users. As Mika (2007) describes it, the Web of the nineties was much like the combination of a phone book and the yellow pages, a mix of individual postings and corporate catalogues, and instilled a little sense of community among its users.

Social Network Analysis is a very relevant technique that has emerged in modern sociology, and which studies the interaction between individuals and organizations. See Scott and Carrington (2011) and Wasserman and Faust (1995) for the theoretical basis and key techniques in social networks.

The idea of 'social network' was loosely used for over a century to connote complex sets of relationships between members of social systems at all scales, from interpersonal to international (Freeman 2004). In 1954, J. A. Barnes used the term systematically to denote patterns of ties, and is normally considered the father of that expression. However, the visual approach to measuring social relationships using graphs, known as sociograms, was presented by Jacob Moreno (1934). In Moreno's network, the nodes represent individuals, while the edges stand for personal relationships. This scientific area of sociology tries to explain how diffusion of innovation works, why alliances and conflicts are generated in groups, how the leadership emerges and how the group structure affects the group efficacy (Mika 2007). 
A major development on the structure of social networks came from a remarkable experiment by the American psychologist Stanley Milgram (Milgram 1967). Milgram's experiment consisted in sending letters from people in Nebraska, in the Midwest, to people in Boston, on the East Coast, where the latter were instructed to pass on the letters, by hand, to someone else they knew. The letters that reached the destination were passed by around six people. Milgram concluded that the experiment showed that, on average, Americans are no more than six steps away from each other. This experiment led to the concepts of the six degrees of separation and the notion of smallworld.

An interesting example of a small-world is the 'Erdös Number' (Grossman et al., 2007). Erdös is the most prolific mathematician, being author of more than 1500 papers with more than 500 co-authors. Erdös is the number zero and the researchers who worked with him are called Erdös number 1. The co-authors of Erdös number 1 are the Erdös number 2, and so on, building one of the oldest small-world known. The work of Erdös and Renyi (1959) describes interesting properties of random graphs. A brand new interest has been revived with the Watts and Strogatz (1998) model, published in the Nature journal, which studies graphs with small-world properties and powerlaw degree distribution.

The social network analysts need to survey each person about their friends, ask for their approval to publish the data and keep a trace of that population for years. Also, the applications, implemented on internet, that uses the concept of establishing links between friends and friends of friends, like Facebook or Linkedln (Linkedln Corporation), provide the required data. According to Linton Freeman's comprehensive Development of Social Network Analysis, the key factors defining the modern field of social network analysis are: the insight that the structure of networks affects the outcome of aggregate actions, and the methodological approach that uses systematic empirical data, graphic representation, and mathematical and computational models to analyze networks. These attributes of social network analysis were established through the work of scientists from the fields of psychology, anthropology, and mathematics over the last decades (Freeman 2004). 
The visualization of a small number of vertices can be completely mapped. However, when the number of vertices and edges increases, the visualization becomes incomprehensible. The large amount of data extracted from the Internet is not compatible with the complete drawing. There is a pressing need for new pattern recognition tools and statistical methods to quantify large graphs and predict the behavior of network systems.

Graph mining can be defined as the science and the art of extracting useful knowledge, like patterns and outliers provided, respectively, by repeated and sporadic data, from large graphs or complex networks (Faloutsos et al., 1999; Cook and Holder, 2007). As these authors put it, there are many differences between graphs; however, some patterns show up regularly, the main ones appearing to be: the small worlds, the degree distribution and the community mining.

In this chapter, the clique communities are studied using the graph partition approach, based on the k-clique structure. A k-clique is a relaxed clique, i.e., a k-clique is a quasi-complete sub-graph. A k-clique in a graph is a sub-graph where the distance between any two vertices is no greater than $\mathrm{k}$. It is a relevant structure to consider when analyzing large graphs like the ones arising in social network analysis.

The proposed Socratic questioning is the following: How many k-clique communities are needed to cover the whole graph? This work is part of a larger project on common knowledge of proverbs whose previous results were published in Mendes, Funk, Cavique (2010).

\section{Graph Theory Concepts}

The representation of social networks has been quite influenced by graph theory. In the social networks, the set of vertices (or nodes) correspond to the "actors" (i.e. people, companies, social actors) and the set of edges to the "ties" (i.e. relationships, associations, links).

The sociologic applications of cohesive subgroups can include groups such as work groups, sport teams, political party, religious cults, or hidden structures like criminal gangs and terrorist cells. In this section, some concepts about cohesive subgroups like cliques and relaxed cliques, such as k-clique, kclub/k-clan and k-plex, are explained. 


\subsection{Graph notation}

Graph theory has many applications and has been used for centuries. The book by Berge (1958), called "Théorie des Graphes e ses Aplications", published many of the knowledge known at the time. A latter edition, in 1973, established a very common notation in graph theory literature that is also used in this chapter.

In this notation, an undirected graph is represented by $G=(V, A)$, where $\mathrm{A} \subseteq[\mathrm{V}]^{2}$ is a pair in which $\mathrm{V}(\mathrm{G})$ represents the set of vertices or nodes, and $A(G)$, the set of links or edges. An edge can be also represented by $\{i, j\} \in A(G)$, where $i$ and $j$ are the two connected vertices. The number of vertices $V(G)$ can be represented by $|V(G)|$ and the graph called of order $n$ if $V(G)=\{1,2, \ldots, n\}$ and so, $|V(G)|=n$. The number of arcs $m$ is given by the cardinality of $A(G)$, i.e. $|A(G)|$. If two vertices are joined by an edge, they are adjacent.

A graph $G^{\prime}=\left(V^{\prime}, A^{\prime}\right)$ is a sub-graph of the graph $G=(V, A)$ if $V^{\prime} \subseteq V$ and $A^{\prime} \subseteq A$. We can also say that if $C$ is a proper subset of $V$, than $G^{\prime}=G-C$ denotes the sub-graph induced from $G$ by deleting all vertices in $C$ and their incident edges. In Figure 1. the graph G' is a sub-graph induced by G, while G" is not, as only edges are missing.

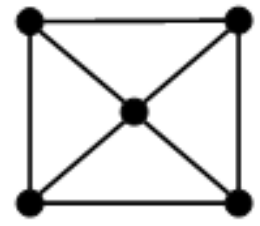

G

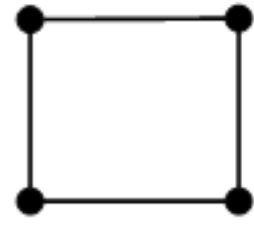

G'

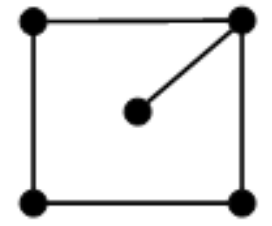

G”

Figure 1 Graph G and two sub-graphs G' and G”.

In Social Network Analysis, the order of the end-vertices of an edge is usually irrelevant and so, we have to work only with undirected graphs. In directed graphs, each directed edge (usually, called arc), has an origin and a destination, and is represented by an ordered pair. In social network contexts, the direction of an edge is not relevant; what is important is to acknowledge the existence, or not, of a link between the edges. 


\subsection{Clique}

Given an undirected graph $\mathrm{G}=(\mathrm{V}, \mathrm{E})$, where $\mathrm{V}$ denotes the set of vertices and $E$, the set of edges, the graph $G_{1}=\left(V_{1}, E_{1}\right)$ is called a sub-graph of $G$, if $V_{1} \subseteq V, E_{1} \subseteq E$ and for every edge $\left(v_{i}, v_{j}\right) \in E_{1}$, the vertices $v_{i}, v_{j} \in V_{1} . A$ sub-graph $G_{1}$ is said to be complete, if there is an edge for each pair of vertices. In fact, a clique is a complete sub-graph, which means that in a clique, each member has direct ties with each other member or node. Some simple examples of these very cohesive structures are shown in Figure. A clique is maximal, if it is not contained in any other clique. The clique number of a graph is equal to the cardinality of the largest clique of $\mathrm{G}$ and it is obtained by solving the maximum clique NP-hard problem.

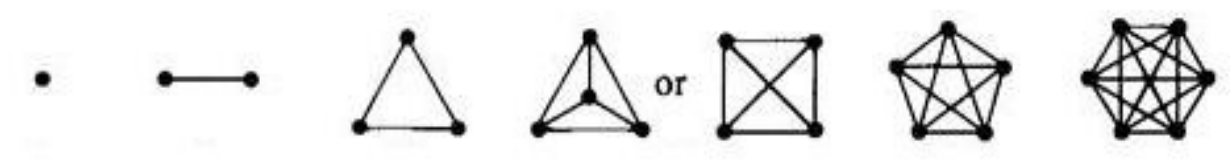

Figure 2 Cliques with 1, 2, 3, 4, 5 and 6 vertices.

The clique structure, where there must be an edge for each pair of vertices, shows many restrictions in real life modeling and is uncommon in social networks. So, alternative approaches for little more relaxed cohesive groups were suggested, such as k-clique, k-clan/k-club and k-plex.

\section{3 k-clique}

Luce (1950) introduced the distance base cohesion groups called kclique, where $\mathrm{k}$ is the maximum path length between each pair of vertices. $A$ k-clique is a subset of vertices $C$ such that, for every $i, j \in C$, the distance $d(i, j)$ $\leq \mathrm{k}$. The 1-clique is identical to a clique, because the distance between the vertices is one edge. The 2-clique is the maximal complete sub-graph with a path length of one or two edges. The path distance of two can be exemplified by the "friend of a friend" connection in social relationships. In social websites, like the Linkedln, each member can reach his own connections as well as the 
ones two and three degrees away. The increase of the value $\mathrm{k}$ corresponds to a gradual relaxation of the criterion of clique membership. See Figure 2.

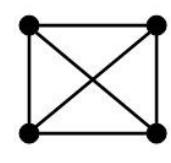

1-clique

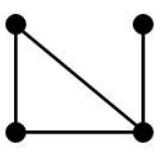

2-clique

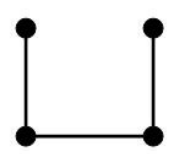

3-clique

Figure 3 Examples with four nodes of 1-clique, 2-clique and 3-clique.

\section{$2.4 \quad k$-clan and k-club}

A limitation of the k-clique concept is that some vertices may be distant from the group, i.e. the distance between two nodes, may correspond to a path involving nodes that do not belong to the k-clique. To overcome this handicap Alba (1973) and Mokken (1979) introduced the diameter-based cohesion group concepts called k-club and k-clan. The length of the shortest path between vertices $u$ and $v$ in $G$ is denoted by the distance $d(u, v)$. The diameter of $G$ is given by $\operatorname{diam}(G)=\max d(u, v)$ for all $u, v \in V$. To find all $k$-clan, all the $k$ cliques $S i$ must be found first, and then the restriction diam(G[S]) $\leq k$ applied to remove the undesired k-cliques. In Figure 3 , on the left, the 2-clique $\{1,2,3,4,5\}$ was removed because $d(4,5)=3$, i. e. the path $4-6-5$ is not possible as node 6 does not belong to the sub-graph with the 2-cliques. Another approach to these diameter models is the k-club, which is defined as a subset of vertices $S$ such that $\operatorname{diam}(G[S]) \leq k$. In the left graph of Figure 3, can be found two 2cliques: $\{1,2,3,4,5\}$ and $\{2,3,4,5,6\}$, one 2-clan: $\{2,3,4,5,6\}$ and three 2-clubs: $\{1,2,3,4\},\{1,2,3,5\}$ and $\{2,3,4,5,6\}$.
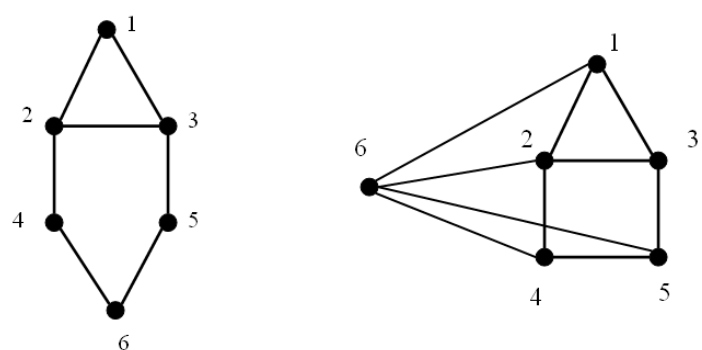

Figure 4 2-clans, 2-clubs (left) and 3-plex (right). 


\section{$2.5 \quad$ k-plex}

An alternative way of relaxing a clique is the k-plex concept which takes into account the vertices degree. The degree of a vertex of a graph is the number of edges incident on the vertex, and is denoted by $\operatorname{deg}(\mathrm{v})$. The maximum degree of a graph $G$ is the maximum degree of its vertices and is denoted by $\Delta(\mathrm{G})$. On the other hand, the minimum degree is the minimum degree of its vertices and is denoted by $\delta(G)$. A subset of vertices $S$ is said to be a k-plex, if the minimum degree in the induced sub-graph $\delta(G[S]) \geq|S|-k$. In Figure, on the right, the graph has 6 vertices and so, $|S|=6$ and the degree of vertices 1, 3, 4 and 5 does not exceed the value 3 . Thus, the minimum degree in the induced sub-graph $\delta(G[S])$ is 3 . For $|S|=6, k=3$ is obtained.

\section{The Two Phase Algorithm}

Complex network and graph mining metrics are essentially based on low complexity computational procedures, like the diameter of the graph, the degree distribution of the nodes and connectivity checking, underestimating the knowledge of the graph structure components.

On the other hand, in the literature, many algorithms have been developed for network communities. One of the first studies is given by the Kernighan, Lin (1970) algorithm, which finds a partition of the nodes into two disjoint subsets $A$ and $B$ of equal size, such that the sum of the weights of the edges between nodes in $A$ and $B$ is minimized. Recent studies, based on physics method, introduced the concept of clique percolation (Derenyi, Palla, Vicsek 2005), where the network is viewed as a union of cliques.

In order to find the k-clique communities, a two-phase algorithm is proposed. First, all the maximal k-cliques in the graph are found. Second, the best subset of the k-cliques is chosen to cover the vertices of the graph.

To find all the maximal $k$-cliques in the graph, we use the $k^{\text {th }}$ power of the graph $G$ in such a way that we can use an already well known algorithm, the maximum clique algorithm. The procedures described in the next flowchart starts by transforming the graph and applying next a maximum clique algorithm and finally, in phase two, applying a set covering algorithm. 
Input: distance $\mathrm{k}$ and graph $\mathrm{G}$

Output: k-clique cover

1. Find all maximal k-cliques in graph $\mathrm{G}$

1.1. The $k^{\text {th }}$ power of graph $G$

1.2. Apply maximum clique algorithm

2. Find the cover of $\mathrm{G}$ with k-cliques

2.1. Apply set covering algorithm

Algorithm 1 - The two-phase algorithm.

\subsection{Maximal k-cliques in graph $\mathbf{G}$}

The transformation of a graph $\mathrm{G}(\mathrm{V}, \mathrm{E})$ into a graph such that for every $i, j \in V$, the distance $d(i, j) \leq k$, is denoted by graph $G(V, E)^{k}$.

The $G(V, E)^{k}$ is obtained using the $k^{\text {th }}$ power of the graph $G$ with the same set of vertices as $G$ and a new edge between two vertices if there is a path of length at most k between them (Skiena 1990).

The Maximum Clique is a NP-hard problem that aims to find the largest complete sub-graph in a given graph. In this approach, we intend to find a lower bound for the maximization problem, based on the heuristics proposed by Johnson (1974) and in the meta-heuristic that uses Tabu Search developed by Soriano and Gendreau (1996). Part of the work described in this section can also be found in Cavique, Rego and Themido (2002) and Cavique and Luz (2009).

We define $A(S)$ as the set of vertices that are adjacent to vertices of a current solution $S$. Let $n=|S|$ be the cardinality of a clique $S$ and $A^{k}(S)$ the subset of vertices with $k$ arcs incident in $S$. $A(S)$ can be divided into subgroups $\mathrm{A}(\mathrm{S})=\cup \mathrm{A}^{\mathrm{k}}(\mathrm{S}), \mathrm{k}=1, \ldots, \mathrm{n}$.

The cardinality of the vertex set $|\mathrm{V}|$ is equal to the sum of the adjacent vertices $A(S)$ and the non-adjacent ones $A^{0}(S)$, plus $|S|$, resulting in $|V|=$ $\Sigma\left|A^{k}(S)\right|+n, k=0, . ., n$. For a given solution $S$, we define a neighborhood $N(S)$ if it generates a feasible solution S'.

In this work we are going to use three neighbourhood structures. For the next flowchart consider the following notation: 


$$
\begin{aligned}
& N^{+}(S)=\left\{S^{\prime}: S^{\prime}=S \cup\left\{v^{i}\right\}, v^{i} \in A^{n}(S)\right\} \\
& N^{-}(S)=\left\{S^{\prime}: S^{\prime}=S \backslash\left\{v^{i}\right\}, v^{i} \in S\right\} \\
& N^{0}(S)=\left\{S^{\prime}: S^{\prime}=S \cup\left\{v^{v}\right\} \backslash\left\{v^{k}\right\}, v^{i} \in A^{n-1}(S), v^{k} \in S\right\}
\end{aligned}
$$

where $S$ is the current solution, $S^{*}$, the highest cardinality maximal clique found so far, $\mathrm{T}$, the tabu list and $\mathrm{N}(\mathrm{S})$, the neighborhood structures.

Input: graph $\mathrm{G}^{\mathrm{k}}$, complete sub-graph $\mathrm{S}$

Output: clique $\mathrm{S}^{*}$

1. $T=\varnothing ; S^{*}=S$;

2. while not end condition

2.1. if $\left(\mathrm{N}^{+}(\mathrm{S}) \backslash T \neq\right.$ null) choose the maximum $\mathrm{S}^{\prime}$

2.2. else if $\left(N^{0}(S) \backslash T \neq\right.$ null $)$ choose the maximum $S^{\prime}$; update $T$

2.2.1. else choose the maximum $\mathrm{S}^{\prime}$ in $\mathrm{N}^{-}(\mathrm{S})$; update $\mathrm{T}$

2.3. update $\mathrm{S}=\mathrm{S}^{\prime}$

2.4. if $\left(|S|>\left|S^{*}\right|\right) S^{*}=S$;

3. end while;

4. return $S^{*}$;

Algorithm 2 - The Tabu Heuristic for the Maximum Clique Problem

Finding a maximal clique in a graph $G^{k}$ is the same as finding a maximal $k$ clique in a graph $\mathrm{G}$. To generate a large set of maximal k-cliques, a multi-start algorithm is used, which calls the Tabu Heuristic for Maximum Clique Problem.

\subsection{The k-cliques Cover}

To understand the structure of a clique community of a network in the previous work (Cavique, Mendes and Santos 2009), the minimum set covering formulation was used.

The detailed analysis of the resulting solution, the set of k-cliques, an excess of over-coverings can be found, which makes it hard to interpret the clique communities. For each pair of k-cliques, the nodes that belong to both $\mathrm{k}$ cliques, are called "bridges" between the two communities. In the next figure, the matrix shows the bridges between the $15 \mathrm{k}$-cliques, with $\mathrm{k}$ equal 3 , for the 
Erdos-97-1 dataset, where the large density of connections does not allow for a clear interpretation of the network.

\begin{tabular}{|l|c|c|c|c|c|c|c|c|c|c|c|c|c|c|c|}
\hline & $\mathbf{1}$ & $\mathbf{2}$ & $\mathbf{3}$ & $\mathbf{4}$ & $\mathbf{5}$ & $\mathbf{6}$ & $\mathbf{7}$ & $\mathbf{8}$ & $\mathbf{9}$ & $\mathbf{1 0}$ & $\mathbf{1 1}$ & $\mathbf{1 2}$ & $\mathbf{1 3}$ & $\mathbf{1 4}$ & $\mathbf{1 5}$ \\
\hline $\mathbf{1}$ & & 128 & 132 & 122 & 122 & 139 & 147 & 123 & 125 & 130 & 138 & 140 & 150 & 144 & 155 \\
\hline $\mathbf{2}$ & & & 151 & 138 & 145 & 158 & 153 & 147 & 142 & 151 & 154 & 160 & 161 & 152 & 161 \\
\hline $\mathbf{3}$ & & & & 173 & 171 & 182 & 181 & 174 & 174 & 180 & 186 & 191 & 194 & 184 & 193 \\
\hline $\mathbf{4}$ & & & & & 181 & 176 & 172 & 188 & 185 & 184 & 194 & 196 & 197 & 197 & 196 \\
\hline $\mathbf{5}$ & & & & & 181 & 170 & 197 & 191 & 186 & 196 & 199 & 193 & 197 & 195 \\
\hline $\mathbf{6}$ & & & & & & & 183 & 181 & 183 & 189 & 196 & 199 & 200 & 191 & 200 \\
\hline $\mathbf{7}$ & & & & & & & & 174 & 180 & 181 & 192 & 192 & 201 & 195 & 206 \\
\hline $\mathbf{8}$ & & & & & & & & & 191 & 192 & 201 & 204 & 197 & 201 & 200 \\
\hline $\mathbf{9}$ & & & & & & & & & & 187 & 206 & 201 & 203 & 204 & 205 \\
\hline $\mathbf{1 0}$ & & & & & & & & & & & 203 & 209 & 202 & 197 & 203 \\
\hline $\mathbf{1 1}$ & & & & & & & & & & & & 216 & 217 & 215 & 219 \\
\hline $\mathbf{1 2}$ & & & & & & & & & & & & & 220 & 212 & 223 \\
\hline $\mathbf{1 3}$ & & & & & & & & & & & & & & 222 & 231 \\
\hline $\mathbf{1 4}$ & & & & & & & & & & & & & & & 226 \\
\hline $\mathbf{1 5}$ & & & & & & & & & & & & & & & \\
\hline
\end{tabular}

Figure 5 Bridges between the 15-set of k-cliques in the k3-Erdos-97-1 dataset

The minimum set covering algorithm generates $15 \mathrm{k}$-cliques, which covers all the 283 nodes, but over-covering 252 nodes.

In this paper, we propose a trade-off between the covered and overcovered nodes. The new metric finds the best solution when the number of covered nodes does not exceed the number of over-covered ones. In other words, the best solution is found when the difference between covered and over-covered nodes is maximal. 


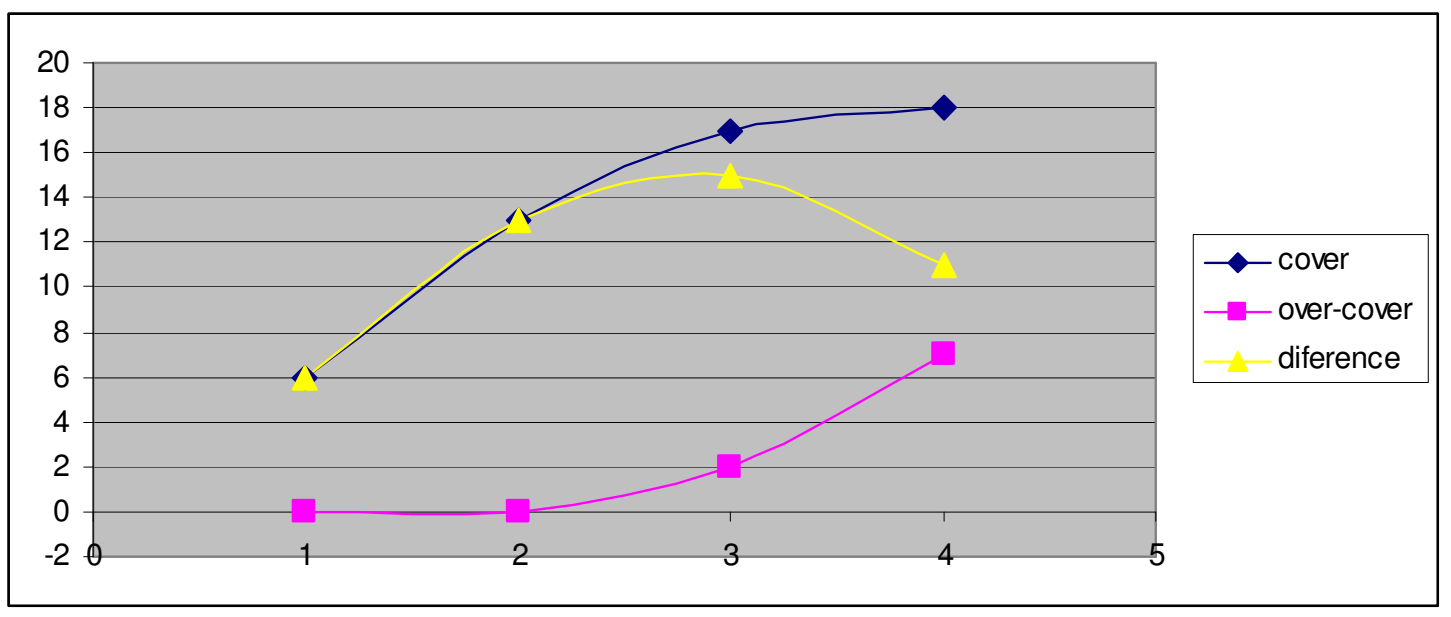

Figure 6 Best trade-off solution happens when the difference is maximal

The k-clique cover algorithm implementation is composed of a constructive step and a reduction step.

The input for the k-clique cover is a matrix where each line corresponds to a node of the graph and each column, a k-clique covering a certain number of nodes.

In the constructive step, the Clique Cover heuristic, proposed by Kellerman (1973) and improved by Chvatal (1979), is used.

We consider the following notation: $M$ [line, column] or M [vertex, kclique] for the input matrix, $C$ for the cost vector of each column, $V$ for the vertex set of $G(V, E)$ and $S$ for the set covering solution.

Input: M [line, column], C, V

Output: the cover $\mathrm{S}$

1. Initialize $\mathrm{R}=\mathrm{M}, \mathrm{S}=\varnothing$,

// Constructive Step

2. While $R \neq \varnothing$ do

2.1. Choose the best line $\mathrm{i}^{\star} \in \mathrm{R}$ such as $\left|\mathrm{M}\left(\mathrm{i}^{\star}, \mathrm{j}\right)\right|=\min |\mathrm{M}(\mathrm{i}, \mathrm{j})| \forall \mathrm{j}$

2.2. Choose the best column $\mathrm{j}^{*}$ that covers line $\mathrm{i}^{*}$

2.3. Update $R$ and $S, R=R \backslash M\left(i, j^{*}\right) \forall i, S=S \cup\left\{j^{*}\right\}$

3. End while

4. Sort the cover S by descending order of costs 
5. For each $\mathrm{Si}$ do if (SISi is still a cover) then $\mathrm{S}=\mathrm{S} \backslash \mathrm{Si}$

// Reduction Step

6. While (over-cover $>$ cover) do

6.1. Choose the column $\mathrm{j}^{*}$ such as (over-cover $>$ cover)

6.2. Remove column $\mathrm{j}^{*}$

7. End While

8. Return S

Algorithm 3 - The Heuristic for the k-clique covering.

In the constructive step, for each iteration, it is chosen a line to be covered and the best column that covers that line. Then, the solution $\mathrm{S}$ and the remaining vertex $R$, are updated. The chosen line is usually the line that is more difficult to cover, i.e. the line that corresponds to fewer columns. After reaching the cover set, the second step is for removing redundancy, by sorting the cover in descending order of cost and checking if each k-clique is really essential.

In the reduction step, the best trade-off solution is found by removing the most over-covered k-cliques, i.e. the k-cliques with a high degree of nodes over-covering.

This heuristic can be improved using a Tabu Search heuristic, by alternating the constructive step with the removal of the most expensive columns, finding a trajectory of solutions, as presented in Gomes, Cavique e Themido (2006).

The solution obtained with the reduction step, decreases the number of k-cliques that covered all the nodes, allowing for a better interpretation of the network. The sub-covered (or not-covered) nodes are treated as outlier nodes and thus not considered in the clique community analysis.

In order to get a better interpretability of the network data, this analysis considers the k-cliques covered nodes as communities, the over-covered nodes, as bridges between the communities and the not-cover nodes, as outlier (or marginal) nodes. 


\subsection{Two numeric examples}

In this section, two numeric examples will be presented to show the constructive and the reduction steps.

To exemplify the constructive step, given a graph with 5 vertices and 4 edges with $E=\{(1,2),(2,3),(3,4),(4,5)\}$, the second power of the graph, $k=2$, a new graph with 5 vertices and 7 edges is obtained with $k-E=\{(1,2),(1,3),(2,3)$, $(2,4),(3,4),(3,5),(4,5)\}$.
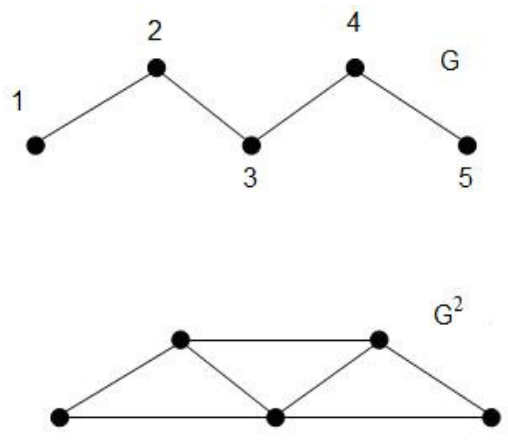

Figure 7 Example of a graph $G$ and its transformation into $a G^{2}$.

Running a multi-start algorithm with the maximum clique problem, three maximal cliques of size 3 can be easily identified: $(1,2,3),(2,3,4)$ and $(3,4,5)$.

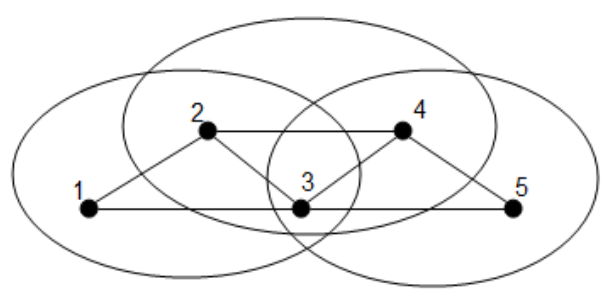

Figure 8 k-clique generation example.

Finally, running the k-cliques cover, in the constructive step of phase 2, two subgroups are found that cover all the vertices. The 2-clique cover is equal to two. Notice that the vertex number 3 appears in the two sets. In social 
network analysis, this is called a "bridge". Indeed, node 3 , with distance 2 can reach any other vertex.

\begin{tabular}{|c|c|c|c|c|}
\hline nodes & $\mathrm{C}$ 1 & $\mathrm{C} 2$ & $\mathrm{C} 3$ & over covered \\
\hline $\begin{array}{c}\text { active } \\
\text { columns }\end{array}$ & 1 & 0 & 1 & \\
\hline 1 & 1 & & & \\
\hline 2 & 1 & 1 & & \\
\hline 3 & 1 & 1 & 1 & 1 \\
\hline 4 & & 1 & 1 & \\
\hline 5 & & & 1 & \\
\hline
\end{tabular}

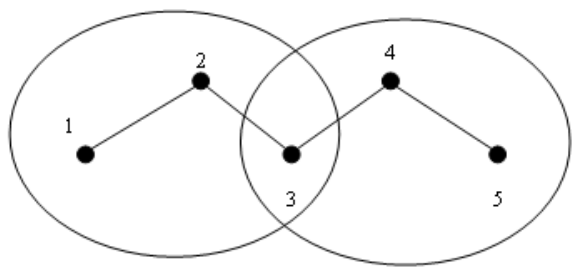

Figure 9 2-sets of 2-cliques cover the whole graph.

The previous figure presents the two subsets solution, using a matrix representation and a graph. For large graphs and a large number of subsets, the graph visualisation gets worse. In these cases, a better general view is attained, using the matrix representation, which is the output of the set covering heuristic.

To show the reduction step of phase 2, let us use a graph with 18 nodes that has a diameter equal to 6 . To cover the whole graph with 3-clique, 3-sets are needed.

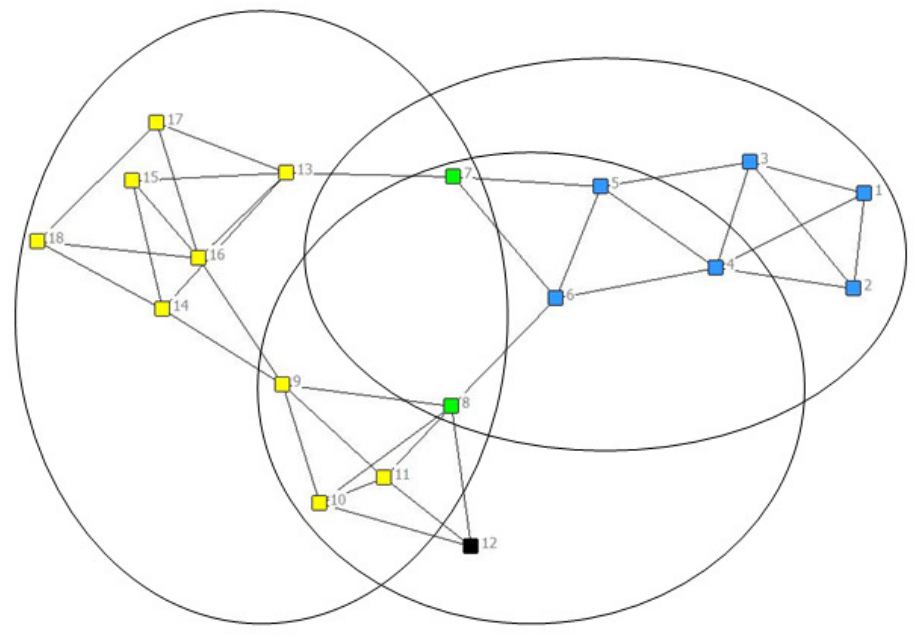

Figure 103 -sets of 3-clique are needed to cover the graph 
The result of the constructive step is 3-sets/columns of 3-clique. In the reduction step, the columns with a larger difference between the covered nodes and the non-covered nodes, will be removed. In the example, one column will be removed, and the final result is a 2-set of 3-cliques, with 2 nodes as bridges (7 and 8) and one marginal node, the node 12 .

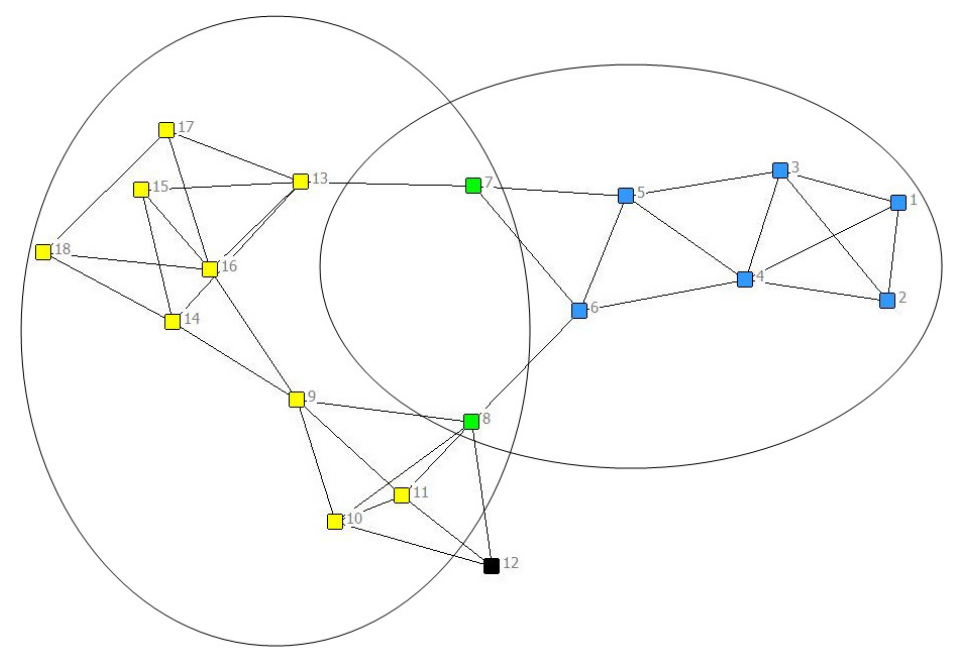

Figure 112 -sets of 3-clique are needed to cover the graph

\section{Applying the algorithm to actual data sets}

To validate the two-phase algorithm, two groups of datasets were used, the Erdös graphs and some clique DIMACS (1995) benchmark instances. In the Erdös graphs, each node corresponds to a researcher, and two nodes are adjacent if the researchers published together. The graphs are named "ERDOS- $x-y$ ", where " $x$ " represents the last two digits of the year that the graphs were created, and "y", the maximum distance from Erdös to each vertex in the graph. The second group of graphs contains some clique instances from the second DIMACS challenge. These include the "brock" graphs, which contain cliques "hidden" within much smaller cliques, making it hard to discover cliques in these graphs. The "c-fat" graphs are a result of fault diagnosis data.

For the analysis of each graph, we consider the number of nodes, the diameter and the cardinality of the set of k-cliques in the constructive and reduction steps, varying $\mathrm{k}$ from 1 to the diameter, as showed in Table 1. 
Table 1 Sequence of k-clique covers in the constructive step and reduction step

\begin{tabular}{|c|c|c|c|c|c|c|c|c|c|c|c|c|}
\hline \multirow[t]{2}{*}{ graph } & \multirow{2}{*}{$\begin{array}{c}\mathrm{nr} \\
\text { nodes }\end{array}$} & \multirow{2}{*}{ diameter } & \multicolumn{10}{|c|}{$\begin{array}{c}\text { cardinality of the k-clique cover } \\
\text { (constructive step; reduction step) }\end{array}$} \\
\hline & & & $\mathrm{k}=1$ & $\mathrm{k}=2$ & $k=3$ & $k=4$ & $k=5$ & $k=6$ & $\mathrm{k}=7$ & $k=9$ & $k=18$ & $\mathrm{k}=40$ \\
\hline test & 18 & 6 & $8 ; 7$ & $4 ; 3$ & $3 ; 2$ & $2 ; 1$ & $2 ; 1$ & $1 ; 1$ & -- & -- & -- & -- \\
\hline erdos-97-1 & 472 & 6 & $9 ; 4$ & $8 ; 1$ & $15 ; 1$ & $10 ; 3$ & $4 ; 3$ & $1 ; 1$ & -- & -- & -- & -- \\
\hline erdos-98-1 & 485 & 7 & $8 ; 4$ & $10 ; 1$ & $12 ; 1$ & $9 ; 3$ & $1 ; 1$ & $1 ; 1$ & $1 ; 1$ & -- & -- & -- \\
\hline erdos-99-1 & 492 & 7 & $8 ; 4$ & $11 ; 1$ & $12 ; 1$ & $9 ; 3$ & $1 ; 1$ & $1 ; 1$ & $1 ; 1$ & -- & -- & -- \\
\hline brock200_1 & 200 & 2 & $24 ; 4$ & $1 ; 1$ & -- & -- & -- & -- & -- & -- & -- & -- \\
\hline brock200_2 & 200 & 2 & $26 ; 9$ & $1 ; 1$ & -- & -- & -- & -- & -- & -- & -- & -- \\
\hline brock400_1 & 400 & 2 & $26 ; 5$ & $1 ; 1$ & -- & -- & -- & -- & -- & -- & -- & -- \\
\hline brock400_2 & 400 & 2 & $23 ; 4$ & $1 ; 1$ & -- & -- & -- & -- & -- & -- & -- & -- \\
\hline c-fat200-1 & 200 & 18 & $28 ; 16$ & $26 ; 10$ & $23 ; 7$ & $20 ; 7$ & $15 ; 5$ & $13 ; 5$ & $12 ; 4$ & $10 ; 4$ & $1 ; 1$ & -- \\
\hline c-fat200-2 & 200 & 9 & $15 ; 10$ & $11 ; 7$ & $7 ; 5$ & $4 ; 4$ & $4 ; 3$ & $6 ; 2$ & $6 ; 2$ & $1 ; 1$ & -- & -- \\
\hline c-fat500-1 & 500 & 40 & $28 ; 16$ & $26 ; 10$ & $23 ; 8$ & $20 ; 7$ & $18 ; 6$ & $17 ; 5$ & $16 ; 5$ & $14 ; 4$ & $8 ; 2$ & $1 ; 1$ \\
\hline
\end{tabular}

In the table, the cardinality of the k-clique cover shows a significant reduction between the two steps: constructive and reduction steps.

For the Erdos-98-1 and Erdos-99-1, with the diameter of 7, the graphs are covered with only 1 -set of 5 -cliques. These values exemplify the difference between k-cliques and k-clans; these graphs are 5-cliques but not 5-clans because the diameter is equal to seven.

The "brock" graphs, known as hiding cliques, have a diameter equal to 2, and to cover the graph, 1-set of 2-clique is enough. Most of the DIMACS instances present this profile. On the other hand, the "c-fat" graphs have the diameter larger than 7 , generating long sequences of k-clique cover.

In the proposed metric, the sequence of k-clique covers identifies families of graphs and seems to be very promising in social network analysis. The k-clique sequence returns a different pattern for each family of networks. In the next figure, the average k-clique cardinality is shown for the different family graphs. 


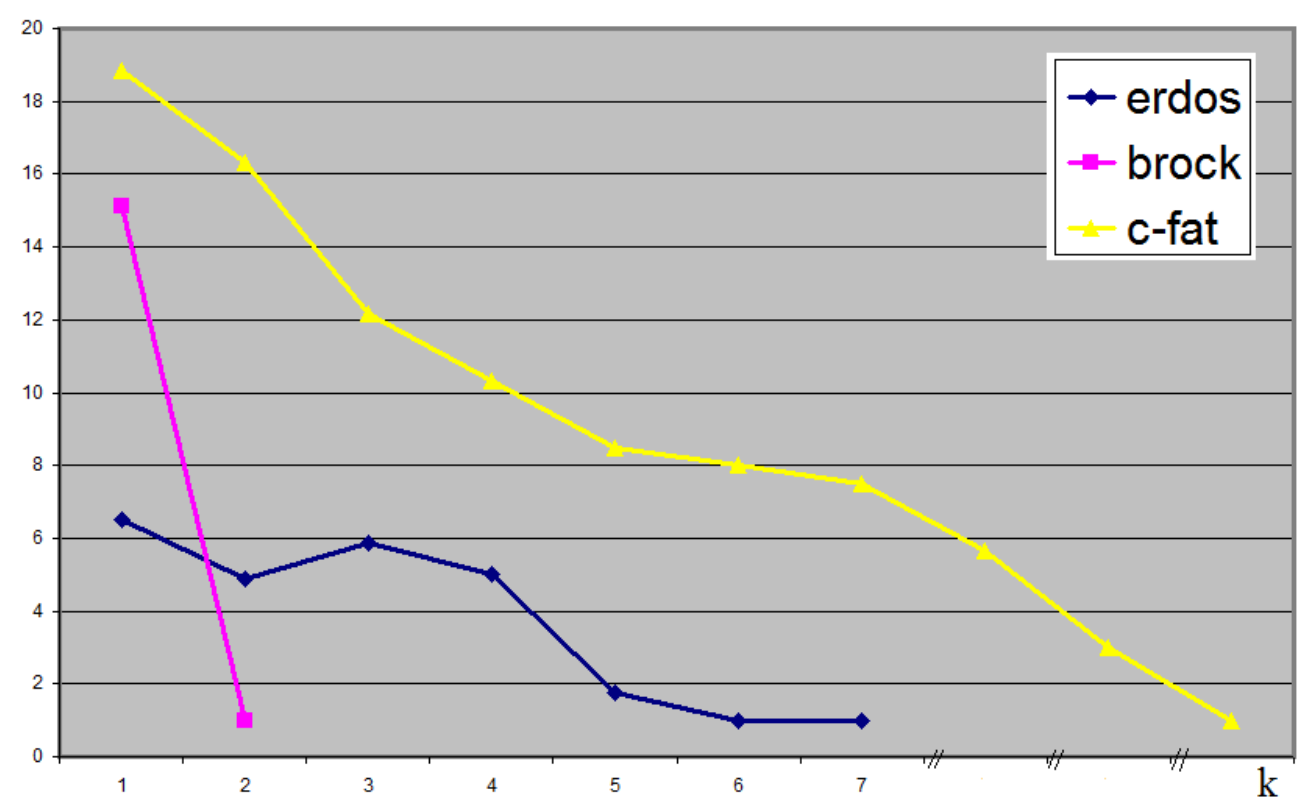

Figure 12 Average k-clique cardinally for the graph families

To answer the initial question about how many k-cliques communities are needed to cover the whole graph, it is done. The social network analyst should choose the best $k$ for his study.

Additional information can be retrieved, like the covered nodes, overcovered nodes and the non-covered nodes, as shown in Table 2, the number of nodes in the bridges, exemplified in Figure 13, and the k-clique composition.

Table 2 Detailed information

\begin{tabular}{|lr|}
\hline File k1-brock400_2 \\
\hline Columns & 4 \\
\hline Total number of lines & 400 \\
\hline Covered lines & 50 \\
\hline Over-covered lines & 13 \\
\hline Non-covered lines & 66 \\
\hline Empty lines & 271 \\
\hline
\end{tabular}




\begin{tabular}{|l|l|l|l|l|}
\hline & $\mathbf{1}$ & $\mathbf{2}$ & $\mathbf{3}$ & $\mathbf{4}$ \\
\hline $\mathbf{1}$ & & 4 & 4 & 5 \\
\hline $\mathbf{2}$ & & & 5 & 2 \\
\hline $\mathbf{3}$ & & & & 3 \\
\hline $\mathbf{4}$ & & & & \\
\hline
\end{tabular}

Figure 13 Bridges between the 4-set of k-cliques in the k1-brock400_2 dataset

\section{Conclusions}

Given the large amount of data provided by the Web 2.0, there is a pressing need to obtain new metrics to better understand the network structure; how their communities are organized and the way they evolve over time.

Complex network and graph mining metrics are essentially based on low complexity computational procedures like the diameter of the graph, clustering coefficient and the degree distribution of the nodes. The connected communities in the social networks have, essentially, been studied in two contexts: global metrics like the clustering coefficient and the node groups, such as the graph partitions and clique communities.

In this work, the concept of relaxed clique is extended to the whole graph, to achieve a general view, by covering the network with k-cliques. A graph mining metric based on k-clique communities, allows for a better understanding of the network structure.

In order to get a good interpretability of the network data, this analysis considers the k-clique covered nodes as communities, the over-covered nodes as bridges between the communities and the not-covered nodes as outlier nodes. The k-clique cover algorithm implementation is composed of a constructive step and a reduction step.

The sequence of k-clique communities is presented, where the diameter and the community structure components are combined. The sequence analysis shows that different graph families have different structures.

Social networks do not usually exceed a hundred nodes. In this work, the proposed two-phase algorithm deals with graphs with hundreds of nodes, with 
a running time performance of a few seconds. Even though this performance may be adequate for practical applications, it is important to study the scalability of the algorithms for much bigger networks like the ones we can find in complex system areas.

With these tools, the social network analyst can measure the basic performance of the networks, study thoroughly the communities of the network by choosing the best $k$ for his/her study.

\section{References}

Alba, R.D. (1973). A graph-theoretic definition of a sociometric clique. Journal of Mathematical Sociology, 3 (3), pp. 113-126.

Berge, Claude (1958). "Théorie des Graphes et ses Applications“. Dunod, Paris.

Berners-Lee, T. (2006) "The Next Wave of the Web" Plenary Panel, 15th International World Wide Web Conference, WWW2006, Edinburgh, Scotland.

Cavique, L.; A.B. Mendes e J.M.A. Santos (2009), "An Algorithm to Discover the k-Clique Cover in Networks", in Progress in Artificial Intelligence, L. Seabra Lopes et al. (Eds.): EPIA 2009, LNAI 5816, Springer-Verlag Berlin Heidelberg, pp. 363-373.

Cavique, L. and Luz, C.J. (2009). A heuristic for the stability number of a graph based on convex quadratic programming and tabu search. Journal of Mathematical Sciences, 161 (6), pp. 944-955.

Cavique, L.; Rego, C. and Themido, I. (2002). A scatter search algorithm for the maximum clique problem. In Ribeiro, C. e Hansen, P. (Eds.) Essays and Surveys in Meta-heuristics. Kluwer Academic Pubs.: Dordrecht, The Netherlands, pp 227-244.

Chvatal V. (1979). A greedy heuristic for the set-covering problem. Mathematics of Operations Research, 4 (4), pp. 233-235. 
Cook, D.J. e Holder, L.B. (Eds) (2007). "Mining Graph Data“. John Wiley \& Sons. DIMACS (1995). Maximum clique, graph coloring, and satisfiability, Second DIMACS implementation challenge, URL http://dimacs.rutgers.edu/ Challenges/, accessed Mars 2011.

Derenyi I., G. Palla, T. Vicsek, (2005), Clique Percolation in Random Networks, Physical Review Letters, vol. 94(16), pp. 160202.

Erdös, P., Renyi, A. (1959). On Random Graphs. I., Publicationes Mathematicae, 6, pp. 290-297.

Faloutsos, M.; Faloutsos, P. and Faloutsos, C. (1999). On power-law relationships of the Internet topology. In Proceedings of SIGCOMM. pp 251262.

Floyd, Robert W. (1962). Algorithm 97: Shortest Path. Communications of the ACM, 5 (5), pp. 345.

Freeman, Linton C. (2004). The Development of Social Network Analysis: A study in the sociology of science. Empirical Press.

Gomes, M.C.; L. Cavique; I.H. Themido (2006). The crew timetabling problem: An extension of the crew scheduling problem. Annals of Operations Research, 144 (144), pp. 111-132.

Grossman, J.; Ion, P. and Castro, R. D. (2007) "The Erdös number Project", URL http://www.oakland.edu/enp/, accessed Mars 2011.

Johnson D.S. (1974). Approximation algorithms for combinatorial problems. Journal of Computer and Systems Sciences, 9 (9), pp. 256-278.

Kellerman E. (1973). Determination of keyword conflict. IBM Technical Disclosure Bulletin, 16(2), pp. 544-546. 
Kernighan, B.W., Lin, Shen (1970). An efficient heuristic procedure for partitioning graphs, Bell Systems Technical Journal, 49, pp. 291-307.

Luce, R.D. (1950). Connectivity and generalized cliques in sociometric group structure. Psychometrika, 15 (15), pp. 159-190.

Mendes, A.; M. Funk and L. Cavique (2010). Knowledge discovery in the virtual social network due to common knowledge of proverbs. In Weiss, Gary M. and Stahlbock, Robert (Eds.) Proceedings of DMIN'10, 6th ed. CSREA Press: USA, pp 213-219.

Mika, Peter (2007). Social Networks and the Semantic Web. Springer-Verlag.

Milgram, S. (1967). The Small World Problem. Psychology Today, 1 (1), pp. 60-67.

Mokken, R.J. (1979). Cliques, clubs and clans. Quality \& Quantity, 13 (13), pp. 161-173.

Moreno, J. L. (1934). Who Shall Survive? Nervous and Mental Disease Publishing Company, Washington D.C..

Scott, John P. and Carrington, Peter (Eds.) (2011). The SAGE Handbook of Social Network Analysis. Sage Pubs.

Skiena, S. (1990). Implementing Discrete Mathematics: Combinatorics and Graph Theory with Mathematica. Reading, MA: Addison-Wesley.

Soriano P.; Gendreau M. (1996) Tabu search algorithms for the maximum clique, In: Johnson, D.S.; Trick, M.A. (Eds.). Clique, Coloring and Satisfiability, Second Implementation Challenge DIMACS, American Mathematical Society, pp. 221-242. 
Wang N., S. Parthasarathy, K.-L. Tan, A.K.H. Tung (2008), CSV: visualizing and mining cohesive subgraphs, in ACM SIGMOD '08 Proceedings, Vancover, Canada.

Watts, D.J. and Strogatz, S.H. (1998). Collective dynamics of small-world networks. Nature, 393 (393), pp. 409-410.

Wasserman, Stanley e Faust, Katherine (1995). Social Network Analysis: Methods and applications. Cambridge University Press. 